\title{
Hypnotic Hallucination of Body Heating Modifies Splanchnic Circulation: Haemodynamic and Ultrasonographic Pilot Study in Normal Volunteers
}

\author{
Valérie Tikhonoff ${ }^{1,2}$, Marco Senzolo ${ }^{3}$, Antonio M. Lapenta ${ }^{2}$, Paolo Palatini ${ }^{1}$, Edoardo Casiglia ${ }^{1,2^{*}}$ \\ ${ }^{1}$ Department of Medicine, University of Padova, Italy \\ 2Institute Franco Granone, Italian Centre of Clinical and Experimental Hypnosis, Torino, Italy \\ ${ }^{3}$ Department of Surgical and Gastroenterological Sciences, Multivisceral Transplant Unit, University Hospital of Padua, Padua, Italy
}

\begin{abstract}
In previous experience we demonstrated that in highly hypnotizable subjects (highs) it is very easy to produce through hypnotic suggestion of heat a real and measurable increase of temperature and of blood flow in body segments. Aim of the present paper is to ascertain whether this is plausible for splanchnic vessels too.

In 5 healthy volunteers aged $27.4 \pm 4.0$ years, cardiac output, upper limb blood flow, mesenteric diameter, flow and mean flow velocity, and portal vein diameter and flow velocity were measured by bioimpedence, ultrasonography and LASER-Doppler in usual conditions of consciousness (pre-hypnosis) and during hypnotic suggestion of body heat. Following hypnotic body heating, splanchnic vasodilation ( $+59 \%$ in mesenteric blood flow, $p<0.01 ;+16 \%$ in diameter; mean flow velocity $+43 \%$ ) was observed, paralleling upper limb flow, while portal vein diameter decreased by $25 \%$ $(p<0.001)$ and portal vein mean flow by $30 \%(p<0.001)$ probably to maintain a constant pressure in the hepatic district in the context of the so-called hepatic arterial buffer response. As a consequence of abdominal and peripheral vasodilation, $9 \%$ reflex rise in heart rate and $15 \%$ in stroke volume were observed, leading to increase of cardiac index in comparison to baseline $(+16 \%)$. Following reflex hyperdynamic status, systolic blood pressure insignificantly tended to increase from $111.8 \pm 21.9 \mathrm{mmHg}$ to $116.0 \pm 13.7 \mathrm{mmHg}(+6 \%)$.

Hypnotic heating is therefore associated to splanchnic arterial vasodilation, a condition that reminds of other physical hyperdynamic conditions. The mental image of body heating therefore produces real and measurable physical effects that can be detected with the tools of physiologists.
\end{abstract}

Keywords: Hypnosis, physiology, human, mesenteric artery, portal vein, peripheral circulation, cardiac index

\section{INTRODUCTION}

The physical effects of hypnosis represent the concrete realization of mental image (Casiglia, 2008, 2012) and

*Correspondence: edoardo.casiglia@unipd.it

Prof. Edoardo Casiglia, Department of Medicine, University of Padova,

Via Giustiniani, 2, 35128 Padova, Italy

Tel +39-049-8212277, Fax +39-049-8754179

\section{Sleep and Hypnosis}

Journal homepage:

www.sleepandhypnosis.org

ISSN:1302-1192 (Print) 2458-9101 (Online) demonstrate that hypnosis is a real, verifiable and repeatable phenomenon. Realization of mental image plays a role in everyday life, but in hypnosis it becomes crucial, producing the phenomenology that is typical of plastic monoideism (Kaffman, 1981; Casiglia et al., 2012a).

Only a limited number of studies described physical effects following hypnotic suggestions, and very few of them described a control on vessel diameter of flow. For instance, Ronel et al. (2011) tried to vasodilate through non-hypnotic verbal suggestions coronary arteries during coronary angiography, obtaining surprisingly vasoconstrictions. 
Reinhard, Hüsken-Janssen, Hatzmann and Schiermeier (2009) observed increase of umbilical flow during neutral hypnotic under cardiotocography in 6 pregnant women.

For physiologists and cardiologists, the physical effects of heating are quite easy to measure. As demonstrated by Casiglia et al. (2006), vasodilation is local if suggestion is local, systemic if suggestion is systemic. In fact, during suggestion of forearm in warm water we previously recorded local vasodilation with decrease of forearm resistance and increase of forearm blood flow like in real local passive warming, and during suggestion of wholebody in a warm water tub there was a systemic vasodilation with decrease of total peripheral resistance and increase of cardiac index like in real total-body passive warming, while during neutral hypnosis (sham procedure) no haemodynamic variations were observed (Casiglia et al., 2006). Nevertheless, our previous study focused on limbs and on thoracic vessels, and did not examine splanchnic circulation.

Actually, the physiology of abdominal arteries and veins are in general limitedly studied, as methods suitable for the evaluation of profound vessels have been made available only recently with the development of ultrasonographic devices. Echo-Doppler (duplex) techniques now allow not only the real-time measurement of vessel diameter but also the quantification of blood flow. At the same time, LASER-Doppler and bioimpedence methods made more and more easy, for a trained physiological staff, the measurement of peripheral flows and the determination of peripheral vessel resistance, minimizing the risk of errors.

The hypothesis tested in the present pilot study is to ascertain whether or not splanchnic circulation can be modified by hypnotic suggestion of body heating. In particular, aim of the study is to use splanchnic ultrasonography in order to verify the occurrence of mesenteric vasodilation, if any, in response to hypnotic hallucination of warm tub bathing. Mesenteric artery diameter and mesenteric blood flow were measured to this aim. At the same time, peripheral and haemodynamic parameters were also monitored via LASER-Doppler, and bioimpedence to clarify whether such variations, if any, were due to blood redistribution between abdomen and peripheral districts, rather than to modifications of cardiac output.

\section{METHODS}

\section{Participants}

This pilot study included 5 young healthy participants (4 women e 1 man), labeled as highly hypnotizable on the basis of a score $\geq 9$ at the Harvard Group Scale of Hypnotic Susceptibility for Italian people (De Pascalis, Russo, \& Marucci, 2000).

The participants, whose general characteristics are shown in Table 1, had to have physiological and anthropometric characteristics allowing the cardiovascular and ultrasonographic monitoring described below, and to be free from cardiac and hepatic diseases possibly confounding the analysis of data.

Table 1: General characteristics of the study subjects (mean \pm standard deviation).

Anthropometrics

Age (years)

Body mass index $\left(\mathrm{kg} \cdot \mathrm{m}^{-2}\right)$

$27.4 \pm 4.0$

Systemic arterial blood pressure and heart rate

Systolic pressure $(\mathrm{mmHg})$

Diastolic pressure $(\mathrm{mmHg})$

Mean pressure $(\mathrm{mmHg})$

Heart rate (beats $\cdot \mathrm{min}^{-1}$ )

$19.9+2.2$

Central haemodynamics

Stroke volume $(\mathrm{ml})$

Cardiac index $\left(l \cdot \mathrm{min}^{-1} \cdot \mathrm{m}^{-2}\right)$

Peripheral haemodynamics

Forearm arterial flow (ARFU)

$107.4 \pm 6.8$

$70.8 \pm 10.6$

$83.0 \pm 10.5$

$53.0 \pm 6.7$

Splanchnic haemodynamics

Mesenteric arterial diameter $(\mathrm{cm})$

Mesenteric arterial flow $\left(30 \pi \cdot \mathrm{cm}^{2} \cdot \mathrm{s}^{-1}\right)$

Portal diameter $(\mathrm{cm})$

Portal flow velocity $\left(\mathrm{cm} \cdot \mathrm{s}^{-1}\right)$

$41.8 \pm 10.0$

$1.4 \pm 0.3$

$4.2 \pm 1.9$

$0.5 \pm 0.1$

$63.8 \pm 85.2$

$1.0 \pm 0.1$

$17.6 \pm 13.4$

Ethics. The participants were previously defined as eligible for hypnosis on the basis of historical questionnaire, of personal interview with the staff and when necessary of Minnesota Multiphasic Personality Inventory 2 test (Butcher \& Williams, 1989; Hathaway \& McKinley, 1985). This preliminary procedure was performed in order to screen those participants, if any, prone to develop side effects secondary to dissociation and therefore not fit for safe hypnotic induction. 
The research adheres to the principles of the Declaration of Helsinki for Human Rights (1990). All participants gave written informed consent to the procedure, were preliminary and personally informed about the aims, methods and possible risks, and had the opportunity to ask all questions they considered necessary. The Ethics Committee of the University Hospital of Padua approved the protocol.

\section{Materials and Procedure}

Protocol. The research described herein has been conducted according to a intra-subject Latin-square protocol, in which each subject is control of himself/ herself. Nonhypnotic basal conditions were compared to hypnotic suggestion of warm tub bathing, while ultrasonographic measurement of splanchnic vessels and peripheral haemodynamics were monitored. The methods used are described below.

Preliminary setting. In preliminary setting, all participants individually underwent hypnotic induction through verbal suggestions with cues of relax and wellbeing, a method already used with success in previous experiments carried out by our research group (Casiglia et al., 1997a, 2007, 2010, 2012b; Facco et al., 2011; Priftis et al., 2011; Tikhonoff et al., 2012). Through the voice of an expert hypnotist, each participant was guided towards focusing his/her attention on a single idea, excluding any other external or internal stimuli. Hypnotic induction consisted of a brief enumeration coupled with suggestions of general well-being, eyelid heaviness, regular deep breathing, and staring at a point. After spontaneous eyelid closure was obtained, participants were invited to concentrate on their own body from head to foot, whilst a feeling of heaviness and muscular relaxation was being suggested (Kaffman, 1981). According to previous papers published by the same research group (Casiglia et al., 1997a, 2006, 2007, 2010, 2012b; Facco et al., 2011), verification of hypnosis was based on some signals, such as arm levitation, the easing of facial tension, a dropped lower jaw with a slight opening of the mouth, and slowing down of breathing rate. The analysis of these signals enabled the hypnotist to verify the participants were really hypnotized and to maintain or modify this condition by means of continuous appropriate suggestions. The aim of this preparatory procedure was to establish a valid interpersonal relationship between the operator and the participant, in order to favor rapid monoideism on the occasion of the following experimental setting. To reduce the time needed for further inductions, post-hypnotic conditioning was predisposed in all participants during this first phase.

Experimental setting. In a different day, the experimental setting was performed. This way to proceed - similar to that followed in previous protocol by the same research group where haemodynamic peripheral modifications following hypnotic suggestion of warm tub bathing had been detected (Casiglia et al., 2006) - was chosen in order to make that research and this experiment absolutely comparable from a procedural point of view.

During the first 30 minutes, with participant in the supine posture, devices aimed at monitoring peripheral
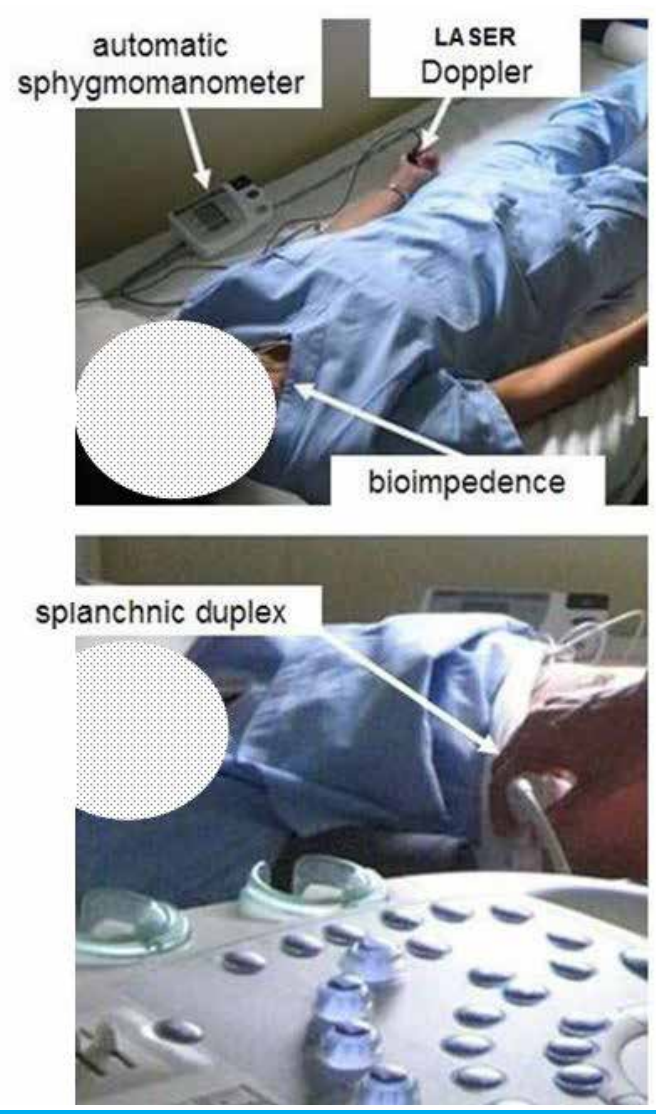

Figure 1: Upper panel: application of devices for measurements of haemodynamic parameters. Lower panel: technique of measurement of splanchnic haemodynamics. 


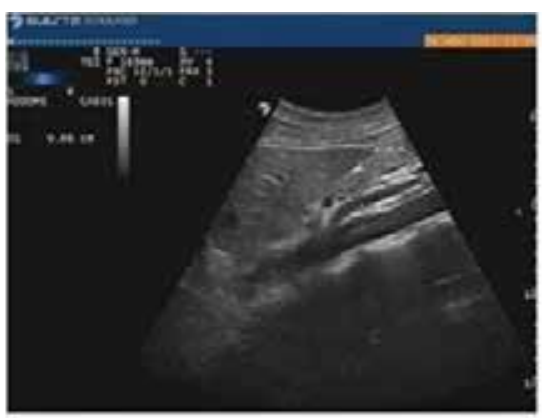

Mesenteric artery diameter

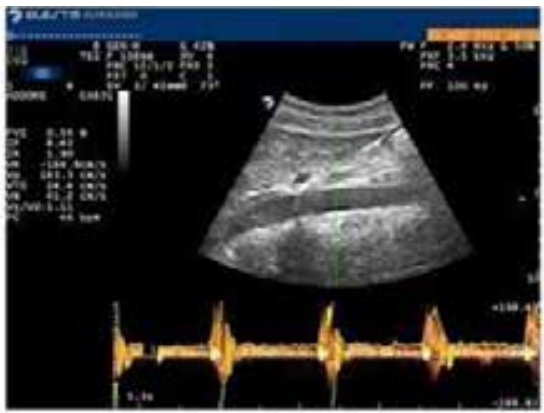

Mesenteric artery flow

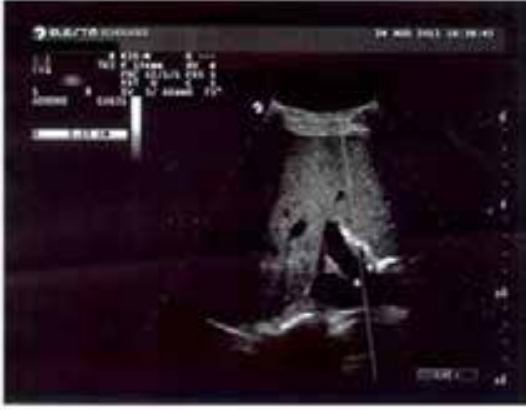

Portal vein diameter

Figure 2: Detection of mesenteric artery diameters and flow and of portal vein diameter in one of the participants (a woman aged 34 years).

haemodynamics were applied as shown in Figure 1 (upper panel). Once haemodynamic stability was reached, echo-Doppler was performed (Figure 1, lower panel) to measure portal vein diameter and flow velocity, and mesenteric artery diameter, flow and mean flow velocity (Figure 2). Baseline measurements of humeral arterial blood pressure, heart rate, forearm arterial flow, stroke volume and cardiac output were also performed as detailed below.

In order to minimize artifacts, all participants were kept, closed-eyes, in the supine position during the entire procedure.

At the end of basal measurements, hypnotic induction was performed via post-hypnotic conditioning through a previously arranged word coupled with handshake. This means that hypnotic induction was immediate and did not require a classic long-lasting induction procedure associated to and dependent on relaxation. Once hypnosis was obtained, special suggestions aimed at obtaining thermal hallucination of body heating were given. The suggestions were similar to those administered in our previous experience, i.e. hallucination of immersion of whole-body in a bath tub of warm water (Casiglia et al., 2006).

At the end of this period, participant's usual consciousness was gradually restored reaching complete dehypnotization.

Anthropometrics. Weight (in kg) and height (in m) were measured without shoes and in light wearing, and body surface area was calculated (in $\mathrm{m}^{2}$ ) from 0.007184 . weigth ${ }^{0.425}$. height $^{0.725}$.

\section{Haemodynamics Measurements}

Splanchnic haemodynamics. Bidimensional ultrasonography was performed by a single examiner (M.S.), according to the guidelines for portal hypertension of the Italian Association for the Study of the Liver (AISF, 2006) through a device equipped with a $3.5 \mathrm{MHz}$ Convex probe used in pulsed Doppler B-mode (My Lab Seven, Esaote, Milan, Italy). Each participant, after staying 30 minutes in the lying posture, underwent suspendedbreathing measurement of the haemodynamic splanchnic parameters listed above. All measurements were repeated 3 times and the values were averaged.

Superior mesenteric artery was visualized in longitudinal section with a subxiphoid view, paralleling aorta and $1.5 \mathrm{~cm}$ from the celiac trunk (Perko, 2001). During a 6-s suspended-breathing period, vessel diameter (in $\mathrm{cm}$ ) and mean flow velocity (in $\mathrm{cm} \cdot \mathrm{s}^{-1}$ ) were measured (Perko, Just, \& Schroeder, 1997). The diameter was taken from the luminal surface of the anterior wall to the luminal surface of the posterior wall and the sample of volume Doppler for the measurement of mean flow rate was placed in the middle of the vessel with an insonation angle $<60^{\circ}$. Blood flow was calculated (in $30 \pi \cdot \mathrm{cm}^{2} \cdot \mathrm{s}^{-1}$ ) from transversal area $\cdot$ mean flow velocity $\cdot 60$, being transversal area equal to $\pi \cdot(1 / 2 \cdot \text { diameter })^{2}$. 
Portal vein was viewed longitudinally through an epigastric approach. During a 6-s suspended-breathing period, portal diameter (in $\mathrm{mm}$ ) and mean flow rate (in $\mathrm{cm} \cdot \mathrm{s}^{-1}$ ) were measured. The diameter was taken from the luminal surface of the anterior wall to the luminal surface of the posterior wall and the sample of volume Doppler for the measurement of mean flow rate was placed in the middle of the vessel, $1-2 \mathrm{~cm}$ before bifurcation, in the tract crossing the hepatic artery, with an insonation angle $<60^{\circ}$.

Systemic parameters. Arterial blood pressure was measured with an Omron 705 IT automatic oscillometric device (HEM-759-E, Omron Corporation, Kyoto, Japan).

Stroke volume (in $\mathrm{ml}$ ) and cardiac index (in $\mathrm{l} \cdot \mathrm{min}^{-1} \cdot \mathrm{m}^{-2}$ ) were measured beat-to-beat through an impedancebased PhysioFlow TM-Lab-1 flowmeter (Manatec Biomedical, Ebersviller, France) (Richard et al., 2001), also giving beat-to-beat heart rate (in beats per minute, $\mathrm{b} \cdot \mathrm{min}^{-1}$ ) calculated from RR intervals (De Benedictis, Cigada, Bianchi, Signorini, \& Cerutti, 1994; Hippel, Hole, \& Kaschka, 2001; Emdin et al., 1996; Sturgis \& Coe, 1990; Ray et al., 2000).

Peripheral haemodynamics. Upper limb arterial flow was measured by an optic-fiber LASER-Doppler flowmeter (Perimed AB, Stockholm, Sweden) (Gooding, Hannemann, Tooke, Clough \& Shore, 2006; Kellogg, 2006). This device records real-time tissue perfusion through a low-power LASER light $(1 \mathrm{~mW})$ focused on thenar eminence of hand. In this technique, the arterial flow (in arbitrary relative flow units, ARFU) is extrapolated from the number and mean velocity of red blood cells moving under the probe.

Statistical analysis. Continuous variables were expressed as mean and standard deviation and compared between groups with one-tailed analysis of variance. Choosing one-tailed analysis was justified by the fact that the expected direction of vessel diameter and flow modifications was known based on previous studies by our research group in which hypnotic hallucination of warm tub bathing was used (Casiglia et al., 2006). The null hypothesis was rejected when $p$ was $<0.05$

\section{RESULTS}

Following hypnotic body heating, splanchnic vasodilation was observed, with $59 \%$ increase in blood flow $(p<0.01)$ in comparison to baseline (Figure 3, upper panel). Mesenteric artery diameter $(0.50 \pm 0.11 \rightarrow 0.58 \pm 0.02 \mathrm{~cm})$ and mean flow velocity $\left(30.4 \pm 4.9 \rightarrow 43.6 \pm 14.1 \mathrm{~cm} \cdot \mathrm{sec}^{-1}\right)$ also tended to increase, although insignificantly. Portal vein diameter significantly decreased by $25 \%(p<0.001)$ and portal vein mean flow velocity by $30 \%(p<0.001)$ (Figure 4 ).

Upper limb blood flow significantly increased by $11 \%$ during hypnotic body heating in comparison to prehypnotic conditions (Figure 3, lower panel).

As a consequence of abdominal and peripheral vasodilation, reflex rise in heart rate (from $53.0 \pm 7.7$ to $57.6 \pm 10.9 \mathrm{~b} \cdot \mathrm{min}^{-1},+9 \%$ ) and stroke volume (from $41.8 \pm 10.0$ to $48.0 \pm 21.3 \mathrm{ml},+15 \%$ ) was observed. Cardiac index therefore tended to increase in comparison to baseline (from $1.38 \pm 0.29$ to $1.60 \pm 0.65 \mathrm{l} \cdot \mathrm{min}^{-1},+16 \%$ ).
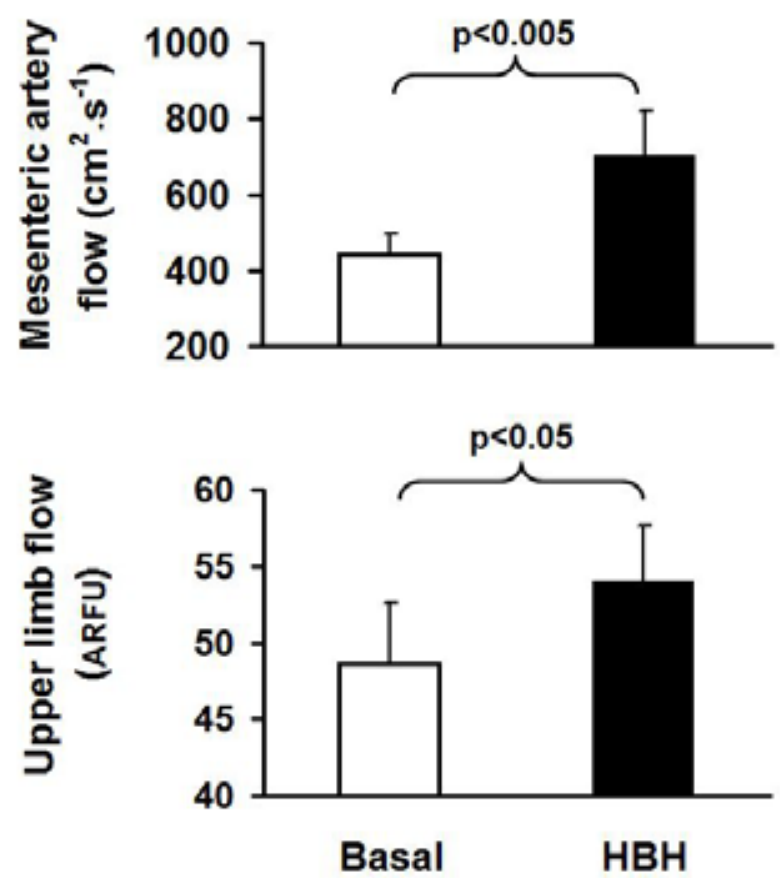

Figure 3: Mesenteric and upper limb arterial flow in non-hypnotic basal conditions and during suggestion of hypnotic body heating (HBH). Mean values and standard deviations are shown. ARFU: arbitrary relative flow units. 

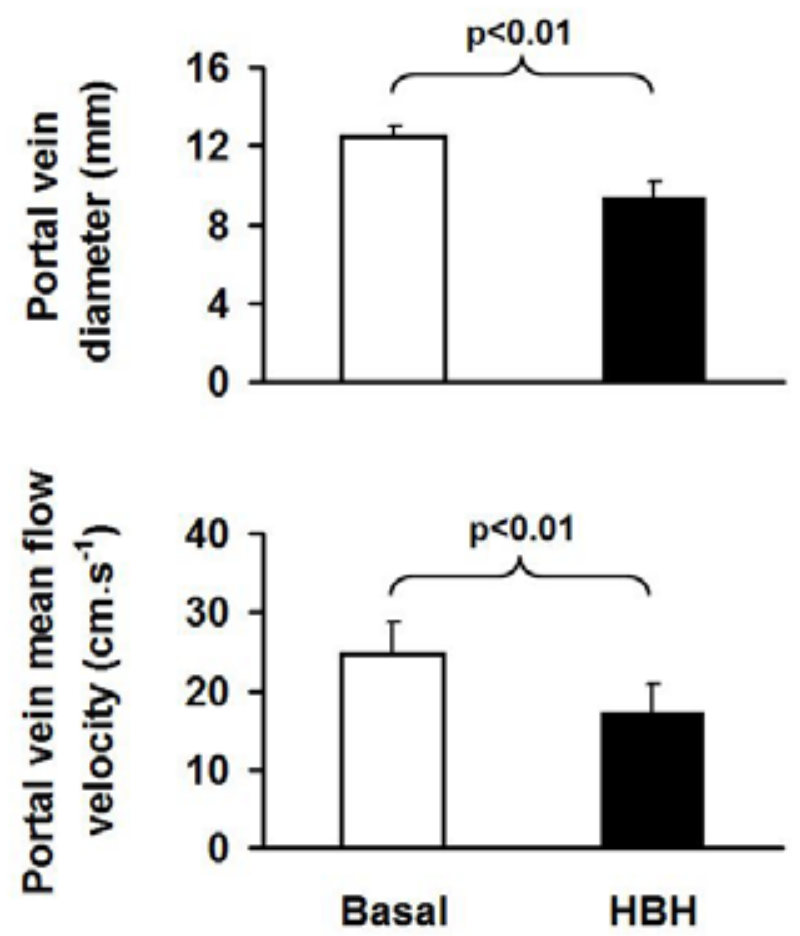

Figure 4: Portal vein diameter and mean flow velocity in non-hypnotic basal conditions and during suggestion of hypnotic body heating (HBH). Mean values and standard deviations are shown.

Following reflex hyperdynamic status, systolic blood pressure insignificantly tended to increase from $111.8 \pm 21.9 \mathrm{mmHg}$ to $116.0 \pm 13.7 \mathrm{mmHg}(+6 \%)$.

\section{DISCUSSION}

During hypnosis, attention is focused monothematically on a particular idea. Although sometimes occurring during normal life, this condition is favoured in the setting of hypnotism by action of an expert operator. In this condition of modified consciousness it is possible to give suggestions that, due to the high level of attention and to the characteristics of modified consciousness, raise to special relevance and can be experienced as belonging to real world and perceived as hallucination (Kaffman, 1981; Rhodes \& Rhodes, 2003; Bryant \& Mallard, 2005). Experiments by our research group previously demonstrated that these hallucinations are not merely subjective, but are accompanied by real and measurable physiological modifications (Casiglia et al., 1997a, 2006; Tikhonoff et al., 2012).

This is particularly true for the sense of heat, a coenaesthesic hallucination that was chosen in this study because it is simple to obtain and because its cardiovascular consequences are easy to measure (Casiglia et al., 2006). Subjective sense of heat is taken for granted even during neutral hypnosis and bio-feedback (Charkoudian, 2003; Herzfeld \& Taub, 1997; Wallace \& Kokoszka, 1992), but is particularly evident when thermal suggestions of heating are given in the setting of hypnotism.

In previous experiments we demonstrated that hypnotic thermal suggestions are accompanied by peripheral vasodilation (Casiglia et al., 2006) like in real warm tub bathing and occur even in the absence of changes in body temperature (Kenny, Giesbrecht \& Thoden, 1996). Hypnotic hallucination of heat is therefore a real and repeatable phenomenon, that can be studied with the experimental models and the techniques typical of human physiology.

In the present study we decided to examine the splanchnic district, that had been disregarded in our previous research. The experimental paradigm was the same used in 2006, and the tools were ultrasonographic detectors of flow and diameter applied to mesenteric artery and to portal vein. The results demonstrated that suggestions aimed at inducing thermal hallucination are followed by splanchnic vasodilation similar to that observed during real immersion in warm water. Splanchnic vasodilation was proven by a statistically significant and biologically relevant increase in superior mesenteric artery flow. According to our previous results (Casiglia et al., 2006), peripheral flow also increased, demonstrating that the mesenteric flow rise must be viewed in the frame of systemic vasodilation rather than of blood diversion from body periphery to the abdomen (Casiglia et al., 1998a, 1998b). The paradigm of hypnotic body heating therefore reminds that of hyperthyroidism, where vasodilation occurring both in splanchnic and systemic districts is characterized by an increase of circulating blood mass and of flow velocity secondary to opening of 
arterial-venous shunts (Fazio, Palmieri, Lombardi \& Biondi, 2004; Perko, Perko, Just, Secher, \& Schroeder, 1996). On the contrary, it is opposite to that of physical exercise, where cardiac output increases while splanchnic flow tends to decrease due to diversion of blood from the abdomen to muscular and subcutaneous tissues (Casiglia et al., 1994, 1997b; Perko, Nielsen, \& Skak, 1998; Tikhonoff et al., 1999).

Apart from research, the above mentioned findings demonstrating the possibility to control with hypnotic suggestions the diameter and the flow of systemic and central vessels - are of potential interest in clinical setting too. In fact, they open the road to some hypothetical clinical uses of hypnosis, such as perfusion increase in functionally vasoconstricted districts (e.g. in accidental frostbite), shunt opening in ischaemic areas, and selective bioavailability of special drugs (for instance antineoplastic medicines) to particular organs or tissues. Of course, the here described study is only based on data collected among normal people in a lab of physiology, and these potential uses are for the moment nothing more than a mere hypothesis, needing ad hoc trials in diseased patients.

Portal vein circulation deserves some special considerations. Portal diameter decreased by $25 \%$ during hypnotic body heating, in contrast to ubiquitous vasodilation. This is in agreement with Crandall et al. (2008), showing a fall in hepatic blood volume during real body heating. The explanation of this finding can only be speculative, as there are practically no previous data in this respect. The trend of portal circulation could derive from increased availability of vasoactive substances (such as adenosine) coming from splanchnic district (Jacobson \& Pawlik, 1992) and/or represent a finalized response aimed at maintaining a constant pressure in the hepatic district in the context of the so-called hepatic arterial buffer response (Eipel, Abshagen, \& Vollmar, 2010). In fact, sympathetic stimulations (such as the reflex sympathetic response induced by systemic vasodilation) are probably accompanied by portal vasoconstriction leading to mobilization of one liter blood in less than one minute.

The present study has several limitations, first of all the low number of subjects. This is due to the very demanding protocol, requiring highly hypnotizable participants and the simultaneous presence of many specialists, such as at the least a hypnotist, a cardiologist expert in haemodynamic measurement, a human physiologist, an operator expert in echo-Doppler detection and measurement of abdominal vessel diameters and flow, and some technicians. Low numerosity could have lead to type- 2 error, i.e. labeling as non significant a difference that could be potentially different. Nevertheless, the paper is mainly based on the positive difference between mesenteric flow measured before and during hypnotic hallucination of warm tub bathing with suggestion of body heating, a difference that should not be influenced by type-2 error. Finally, although the results of the study have consequences of potential interest for physicians, the study protocol was only designed for research purposes, so that it is impossible to extend the results to the world of clinical medicine and psychology. This should be considered a pilot piece of research, and other studies with different protocols, based on greater numbers of participants, are necessary to reply to some questions remained unanswered.

In conclusion, hypnotic body heating is not only associated to peripheral vasodilation as previously demonstrated by our research group (Casiglia et al., 2006), but probably also to splanchnic arterial vasodilation, like in real total-body warm tub bathing. This condition reminds other physical hyperdynamic conditions. The mental image of body heating therefore produces real and measurable physical effects that can be detected with the tools of physiologists.

\section{Conflicts of interest}

The authors do not declare any conflict of interest. 
Tikhonoff / Sleep and Hypnosis 2018

\section{References}

41 ${ }^{\text {st }}$ World Medical Assembly. (1990) Declaration of Helsinki: recommendations guiding physicians in biomedical research involving human subjects. Bulletin of the Pan American Health Organization, 24, 606-609.

Bryant, R. A. \& Mallard, D. (2005). Reality monitoring in hypnosis: a real-simulation analysis. International Journal of Clinical and Experimental Hypnosis, 53(1), 13-25.

Butcher, J. N. \& Williams, C. L. (1989). Fondamenti per l'interpretazione del MMPI-2 e del PI-A. Firenze, Italy: Organizzazioni Speciali Giunti.

Casiglia, E. (2008). Hypnosis in the theory of the bicameral mind. The Jaynesian, 2(1), 12-14

Casiglia, E. (2012). Why is it so easy to hypnotize? Contemporary Hypnosis and Integrative Therapy, 29, 309-314.

Casiglia, E., Mazza, A., Ginocchio, G., Onesto, C., Pessina, A.C., Rossi, A., ... \& Marotti, A. (1997a) Haemodynamics following real and hypnosis-simulated phlebotomy. American Journal of Clinical Hypnosis, 40(1), 368-375.

Casiglia, E., Palatini, P., Bongiovì, S., Colangeli, G., Mario, L. \& Pessina, A. C. (1994). Haemodynamics of recovery after strenuous exercise in physically trained hypertensive and normotensive subjects. Clinical Science, 86(1), 27-34.

Casiglia, E., Palatini, P., Ginocchio, G., Biasin, R., Pavan, L. \& Pessina, A. C. (1998a). Leg versus forearm flow: $24 \mathrm{~h}$ monitoring in 14 normotensive subjects and in 14 age-matched hypertensive patients confined to bed. American Journal of Hypertension, 11(2), 190-195.

Casiglia, E., Pavan, L., Marcato, L., Leopardi, M., Pizziol, A., Salvador, P., ... \& Pessina A. C. (1998b) Subjects with obstructive pulmonary disease tend to be chronically vasodilated. Clinical Science, 95(3), 287-294.

Casiglia, E., Pessina, A. C., Bongiovì, S., Michieletto, M., Ginocchio, G., Biasin, R., ... \& Palatini P. (1997b). Central and peripheral hemodynamics during and after long-lasting two-leg exercise in borderline hypertensive males. International Journal of Sports Cardiology, 6, 133-138.

Casiglia, E., Rossi, A., Tikhonoff, V., Scarpa, R., Tibaldeschi, G., Giacomello, M., ... \& Lapenta A. M. (2006). Local and systemic vasodilation following hypnotic suggestion of warm tub bathing. International Journal of Psychophysiology, 62(1), 60-65.

Casiglia, E., Schiavon, L., Tikhonoff, V., Haxhi Nasto, H., Azzi, M., Rempelou, P., ... \& Rossi A. M. (2007). Hypnosis prevents the cardiovascular response to cold pressor test. American Journal of Clinical Hypnosis, 49(4), 255-266.

Casiglia, E., Schiff, S., Facco, E., Gabbana, A., Tikhonoff, V., Schiavon, L., ... \& Amodio, P. (2010). Neurophysiological correlates of post-hypnotic alexia. A controlled study with Stroop test. American Journal of Clinical Hypnosis, 52(3), 219-233.

Casiglia, E., Tikhonoff, V., Giordano, N., Regaldo, G., Facco, E., Marchetti, P., ... \& Amodio P. (2012a). Relaxation versus fractionation as hypnotic deepening: do they differ in physiological changes? International Journal of Clinical and Experimental Hypnosis, 60(3), 241-261

Casiglia, E., Tikhonoff, V., Giordano, N., Regaldo, G., Tosello, M. T., Rossi, A. M., ... \& Facco, E. (2012b). Measured outcomes with hypnosis as an experimental tool in a cardiovascular physiology laboratory. International Journal of Clinical and Experimental Hypnosis, 60(2), 241-261.
Charkoudian, N. (2003). Skin blood flow in adult human thermoregulation: how it work, when it does not, and why. Mayo Clinic Proceedings, 78(5), 603-612.

Crandall, C. G., Wilson, T. E., Marving, J., Vogelsang, T. W., Kjaer, A., Hesse, B. \& Secher, N. H. (2008). Effects of passive heating on central blood volume and ventricular dimensions in humans. Journal of Physiology, 586(1), 293-301.

De Benedictis, G., Cigada, M., Bianchi, A., Signorini, M. G. \& Cerutti, S. (1994). Autonomic changes during hypnosis: a heart rate variability power spectrum analysis as a marker of sympatho-vagal balance. International Journal of Clinical and Experimental Hypnosis, 42(2), 140-152.

De Pascalis, V., Russo, O. \& Marrucci, F. S. (2000). Italian norms for the Harvard Group Scale of Hypnotic Susceptibility, Form A. International Journal of Clinical and Experimental Hypnosis, 48(1), 44-55.

Eipel, C., Abshagen, K. \& Vollmar, B. (2010). Regulation of hepatic blood flow: the hepatic arterial buffer response revisited. World Journal of Gastroenterology, 16(48), 6046-6057.

Emdin, M., Santarcangelo, E. L., Picano, E., Raciti, M., Pola, S., Macerata, A., ... Labbate, A. (1996). Hypnosis effect on RR interval and blood pressure variability. Clinical Science, 91, 36.

Facco, E., Casiglia, E., Masiero, S., Tikhonoff, V., Giacomello, M. \& Zanette, G. (2011). Effects of hypnotic focused analgesia on dental pain threshold. International Journal of Clinical and Experimental Hypnosis, 59(4), 454-468.

Fazio, S., Palmieri, E. A., Lombardi, G. \& Biondi, B. (2004). Effects of thyroid hormone on the cardiovascular system. Recent Progress in Hormone Research, 59, 31-50.

Gooding, K. M., Hannemann, M. M., Tooke, J. E., Clough, G. F. \& Shore, A. C. (2006). Maximum skin hyperaemia induced by local heating: possible mechanism. Journal of Vascular Research, 43(3), 270-277.

Hathaway, S. R. \& McKinley, J. C. (1985). Minnesota Multiphasic Personality Inventory 2 Manual. P. Pancheri, S. Sirigatti (Eds). Firenze, Italy: Organizzazioni Speciali Giunti.

Herzfeld, G. M. \& Taub, E. (1997). Suggestion as an aid to selfregulation of hand temperature. International Journal of Neuroscience, 8(1), 23-26.

Hippel, C. V., Hole, G. \& Kaschka, W. P. (2001). Autonomic profile under hypnosis as assessed by heart rate variability and spectral analysis. Pharmacopsychiatry, 34(3), 11-113.

Italian Association for the Study of the Liver (A.I.S.F.) (2006). A.I.S.F. Guidelines for Treatment of Portal Hypertension, 7-9.

Jacobson, E. D. \& Pawlik, W. W. (1992). Adenosine mediation of mesenteric blood flow. Journal of Physiology and Pharmacology, 43(1), 3-19.

Kaffman, M. (1981). Monoideism in psychiatry: theoretical and clinical implications. American Journal of Psychotherapy, 35(2), 235-243.

Kellogg, D. L. (2006). In vivo mechanisms of cutaneous vasodilation and vasoconstriction in humans during thermoregulatory challenges. Journal of Applied Physiology, 100(5), 1709-1718

Kenny, G. P., Giesbrecht, G. G. \& Thoden, J. S. (1996). A comparison of human thermoregulatory response following dynamic exercise and warm-water immersion. European Journal of Applied Physiology and Occupational Physiology, 74(4), 336-341. 
Perko, M. J. (2001). Duplex ultrasound for assessment of superior mesenteric artery blood flow. European Journal of Vascular and Endovascular Surgery, 21(2), 106-117.

Perko, M. J., Just, S. \& Schroeder, T. V. (1997). Importance of diastolic velocities in the detection of celiac and mesenteric artery disease by duplex ultrasound. Journal of Vascular Surgery, 26(2), 288-293.

Perko, M. J., Nielsen, H. B. \& Skak, C. (1998). Mesenteric, coeliac and splanchnic blood flow in humans during exercise. Journal Physiology, 513: 907-913.

Perko, M. J., Perko, G., Just, S., Secher, N. H. \& Schroeder, T. V. (1996). Changes in superior mesenteric artery Doppler waveform during reduction of cardiac stroke volume and hypotension. Ultrasound in Medicine and Biology, 22(1), 11-18.

Priftis, K., Schiff, S., Tikhonoff, V., Giordano, N., Amodio, P., Umiltà, C. \& Casiglia, E. (2011). Hypnosis meets neurosciences: simulating visuospatial neglect in healthy participants. Neuropsychologia, 49(12), 3346-3350.

Ray, W. J., Sabsevitz, D., De Pascalis, V., Quigley, K., Aikins, D. \& Tubbs, M. (2000). Cardiovascular reactivity during hypnosis and hypnotic susceptibility: three studies of heart rate variability. International Journal of Clinical and Experimental Hypnosis, 48(1), 22-31.

Reinhard, J., Hüsken-Janssen H., Hatzmann, H. \& Schiermeier, S. (2009). Changes in resistance of the umbilical artery, foetal movements and short time variation through clinical hypnosis preliminary results. Zeitschrift für Geburtshilfe und Neonatologie, 213(1), 23-26.
Rhodes, J. D. \& Rhodes, S. N. (2003). Hypnosis works. The Oklahoma Nurse, 48(2), 13.

Richard, R., Ondsdorfer-Wolf, E., Charloux, A., Doutreleau, S., Buchheit, M., Oswald-Mammosser, M., ... \& Londsdorfer, J. (2001). Non-invasive cardiac output evaluation during a maximal progressive exercise test, using a new impedance cardiograph device. European Journal of Applied Physiology, 85(3-4), 202207.

Ronel, J., Mehilli, J., Ladwig, K. H., Blättler, H., Oversohl, N., Byrne, R. A., ... \& Meissne, K. (2011). Effects of verbal suggestion on coronary arteries: results of a randomized controlled experimental investigation during coronary angiography. American Heart Journal, 162(3), 507-511.

Sturgis, L. M. \& Coe, W. C. (1990). Physiological responsiveness during hypnosis. International Journal of Clinical and Experimental Hypnosis, 38(3), 196-207.

Tikhonoff, V., Azzi, D., Boschetti, G., Giordano, N., Rempelou, P. Giacomello, M., ... \& Casiglia, E. (2012). Increase of isometric performance via hypnotic suggestion: experimental study over 10 young healthy volunteers. Contemporary Hypnosis and Integrative Therapy, 29(4), 352-262.

Tikhonoff, V., Palatini, P., Pizziol, A., Vriz, O., Cazzaro, G., Onesto, C., ... \& Casiglia, E. (1999). Haemodynamic and systemic consequences of a $30-\mathrm{km}$ endurance race in altitude. International Journal of Sports Cardiology, 8, 83-93.

Wallace, B. \& Kokoszka, A. (1992). Experience of peripheral temperature change during hypnotic analgesia. International Journal of Clinical and Experimental Hypnosis, 40(3), 180-183. 